\title{
Introduction and overview
}

Treatment paradigms are evolving rapidly in multiple myeloma (MM) due to advances in our understanding of the disease pathology and the development of new therapies. There have been consistent reports of improvements in survival times over the past decade,1-3 particularly in patients aged younger than 45 years. This improvement in survival is due in part to the use of highdose therapy (HDT) and autologous stem cell transplantation (SCT), and to the introduction of novel treatments, such as thalidomide, bortezomib, and lenalidomide, which can be given to patients of all ages, including elderly patients who are ineligible for autologous SCT and for whom HDT regimens often prove toxic. A retrospective study of survival outcomes in patients diagnosed with MM between 1997 and 2006 found a progressive improvement in overall survival (OS) and, notably, patients who had received one or more of the novel therapies (thalidomide, bortezomib, or lenalidomide) survived twice as long as those patients who did not (30.9 versus 14.8 months, respectively; $p<0.001) .1,2$ It is hoped that the use of novel agents will improve the survival of older patients with $\mathrm{MM}$ - a subset of patients in which survival outcomes have scarcely increased over the past decade.3 Despite treatment advances, MM remains incurable, and all patients eventually relapse due to the re-emergence of the tumor from residual disease that cannot be eradicated. However, new treatment approaches can provide disease control for long periods of time and this has shifted the focus of research toward identifying therapies or combination regimens that offer deep, durable remissions which can be tolerated over the long term in order to prolong the time to relapse and extend OS, with the potential to shift the perception of MM from an acute life-limiting illness toward that of a chronic disease.

Lenalidomide is an IMiDs® immunomodulatory compound with a mechanism of action that includes both tumoricidal and immunomodulatory effects.4-8 The articles in this supplement review what is currently known about the effects of lenalidomide on the biology of MM, its clinical efficacy and safety and how this knowledge is being applied to the development of new treatment strategies and novel combination regimens that have the promise to further improve outcomes for patients with MM. In the first article, Dr Mahindra et al. describe current views on MM pathogenesis and how our improved understanding of disease mechanisms is leading to better treatment approaches and new targeted therapies. Topics discussed include the complex pathogenesis of MM, the importance of chromosomal abnormalities in the progression of MM and as prognostic factors, and the role of microenvironmental factors (e.g. bone marrow stromal cells, specific cytokines, and cell adhesion molecules) in determining MM cell growth and survival.

The practical applications of our improved understanding of MM pathology are highlighted, including a proposed new classification system for MM based on genetic and cellular features 9 and the identification of new antimyeloma targets and new targeted therapies that may be able to overcome resistance to existing MM therapies, thereby further improving the prognosis for patients with advanced disease.

Next, Dr Davies and Dr Baz review what is known about the mechanism of action of lenalidomide in MM. Lenalidomide exerts its tumoricidal effects through a number of complex cellular mechanisms, including the disruption of stromal support from the bone marrow, 10,11induction of tumor suppressor genes, and inhibition of oncogenes leading to cell cycle arrest and the activation of caspases that trigger cell death.4-6,12,13 In addition to rapid tumoricidal effects, lenalidomide has immunomodulatory actions, including T-cell and natural-killer-cell activation, and increased 
expression of death effector molecules. These lead to enhanced immune cell function, $7,14,15$ which may explain the beneficial effects of long-term therapy with this agent.16-19 In this review the authors also discuss how a better understanding of the effects of lenalidomide may lead to improvements in its use, for example in combination with low-dose dexamethasone to optimize both tumoricidal and immunomodulatory effects, 6,20 and in the rational design of novel lenalidomide-based combination regimens with other novel agents.

To bring a clinical perspective to this information, Dr Dimopoulos and Dr Terpos review the available clinical data regarding the efficacy and safety of lenalidomide in MM. Clinical evidence from phase III studies shows that lenalidomide treatment offers high response rates and improved time to progression, progression-free survival, and OS in patients with relapsed or refractory MM in combination with dexamethasone,19,21,22 and in patients with newly diagnosed MM in combination with either dexamethasone23,24 or melphalan plus prednisone.25 In patients with relapsed MM, prolonged lenalidomide treatment is well tolerated and may improve the duration of response and extend OS.16,26 The efficacy and tolerability of lenalidomide have also led to its evaluation as continuous therapy after induction treatment17 or HDT-CT18,19 in patients with newly diagnosed MM; initial clinical results indicate that continuous lenalidomide therapy is able to significantly prolong progression-free survival. Lenalidomide in combination with dexamethasone is also effective in delaying the progression of smoldering myeloma to active $\mathrm{MM}$ in patients at high risk of progression, with phase III data indicating good response rates and tolerability.27 Lenalidomide-based combinations are a focus of clinical research due to the high efficacy, good tolerability, and lack of cumulative toxicity associated with lenalidomide.

The final paper in this supplement, by Dr Morgan, reviews the novel combination regimens in development for the treatment of MM. New combination regimens are designed to build upon the success of available treatment regimens, using our increased understanding of MM biology and the new targeted therapies in development to put together drug combinations that have additive or synergistic antitumor effects. There are numerous novel agents in the early phases of clinical evaluation in combination with lenalidomide plus dexamethasone. These include epigenetic agents such as the oral histone deacetylase inhibitors vorinostat or panobinostat;28 bortezomib;29 and other novel proteasome inhibitors, such as carfilzomib30,31 and NPI-0052.32 Other agents in the early stages of clinical evaluation with lenalidomide plus dexamethasone include inhibitors of mammalian target of rapamycin (mTOR),33 Akt,34 cyclin-dependent kinase inhibitors,35

and interleukin- 6 inhibitors.35 Experimental agents such as heatshock protein 90 inhibitors and drugs targeting the cell surface receptors, such as anti-CS1 and anti-CD40 monoclonal antibodies, are also undergoing clinical evaluation.35-37 This series of articles provides a valuable overview of the relationship between MM biology and the efficacy of lenalidomide as the basis of novel treatment approaches. I am confident that the articles in this supplement will provide informative reading for those involved in the management of MM. 